\title{
Multi-criteria Receiver Self-Election Scheme for Optimal Packet Forwarding in Vehicular Ad hoc Networks
}

\author{
R.H. Khokhar, M.A. Ngadi, M.S. Latiff, K.Z. Ghafoor, S. Ali
}

\author{
Rashid Hafeez Khokhar, Md Asri Ngadi \\ Mohammad Shafie Latiff, Kayhan Zrar Ghafoor, Saqib Ali \\ Faculty of Computer Science and Information Systems, \\ Universiti Teknologi Malaysia, 81310 Skudai, Johor, Malaysia \\ hkrashid2@live.utm.my, dr.asri@utm.my, shafie@utm.my, zgkayhan2@live.utm.my, asaqib2@live.utm.my
}

\begin{abstract}
In most of the existing geographical forwarding methods of Vehicular Ad hoc NETwork (VANET), a node periodically sends "hello" messages to determine the positional information of its direct neighbors. Each node stores and maintains more or less accurate information of its direct neighbors in a table. However, due to high mobility vehicles and traffic congestion the stored neighbors information is quickly outdated, failure notification increases significantly, and leading sub-optimal path. Furthermore, the transmission of periodic "hello" messages and table maintenance consume resources, which is not suitable for sensitive VANET. In this paper, we propose a geographical forwarding mechanism based on Multi-criteria Receiver SelfElection (MRSE) scheme to find best next hop without sending the periodic "hello" messages and maintaining neighbors information in the table. The selection of best next hop is based on the multi-criteria waiting function. In this function, the four key parameters including link life time, optimal distance from sender to receiver, optimal transmission range, and received power are determined to enable the next candidate node to make packet forwarding decisions. The simulation results show that the MRSE scheme performs up to $22 \%$ better in terms of packet delivery ratio as compared to some existing schemes. In terms of average delay, MRSE scheme performs best, with as much as $81 \%$ decrease compared to some existing schemes.

Keywords: Vehicular Ad hoc Networks, VANET Routing, Geographical Forwarding, Multi-Criteria Waiting Function.
\end{abstract}

\section{Introduction}

Vehicular networks are emerging as a new promising field of wireless technology, which aims to deploy vehicle-to-vehicle and vehicle-to-infrastructure communications for different applications such as roadway safety, dynamic route planning, mobile sensing, and in-car entertainment. Vehicular Ad hoc NETworks (VANETs) provide true ubiquitous communication networks with great features as these networks are self configurable, infrastructureless, and rapidly deployable. These promising applications and features of VANETs require an efficient routing protocol for vehicleto-vehicle (V2V) and vehicle-to-infrastructure (V2I) communications. Because of the unique characteristics of VANETs i.e., highly dynamic topology, frequently disconnected network, and various communications environments, the traditional mobile ad hoc network (MANET) routing protocols such as AODV [1], DSR [2], OLSR [3] are not suitable in VANET. The main problem in VANET is route instability, leading to frequent route breaking due to high vehicle speeds and traffic congestion in urban environments. The geographical routing protocols such as GPSR [4], GFG [5], GOAFR [6], GPCR [7], GpsrJ+ [8], GeoCross [9], FAST [10]and offer a suitable solution to handle these problems. However, the geographical forwarding in these protocols do not perform well if they cannot find next hop due to high mobility.

Proposed recovery strategies [4,7] of geographical routing protocols in the literature are not as effective in high mobility where the network topology frequently changing. Figure 1 shows that 
route breaks between nodes $S$ and $A$ are due to either high mobility or staleness of neighborhood information. In this case, node $B$ should be used instead of $A$ to forward data packets to destination node $D$. Initially, the route $S \rightarrow A \rightarrow D$ established at time $t$, and the route breaks when node $A$ moves out of the transmission range of node $S$ after time $t+\lambda t$. Despite the better path stability of geographical forwarding methods, these methods still do not perform well in city environments $([11,12])$.

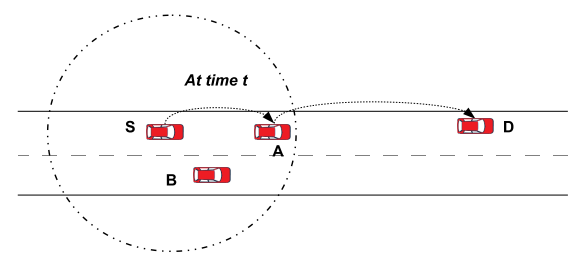

(a) Sending message at time $t$

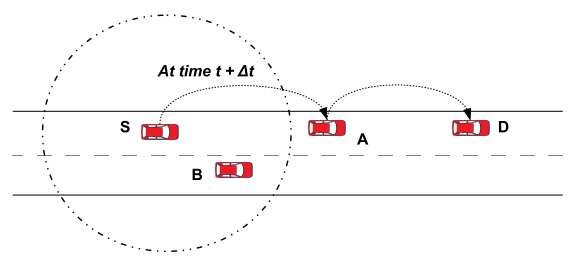

(b) Sending message at time $t+\lambda t$

Figure 1: Frequent route breaking in high mobility using traditional node centric routing protocols

Existing geographical forwarding methods [13-16] use one, two, or three criteria based forwarding scheme to select best next hop. These methods do not consider error-prone wireless channels, low connection time between vehicles, and optimal transmission range. For example, the hop-count based greedy geographic forwarding approaches $[4,17,18]$ has received a great deal of attention in the vehicular ad hoc networking research community. These forwarding approaches have shortcomings due to sub-optimality of packet forwarding, a transmitter tends to select node with poor link quality. As a result, many data packets are dropped and the overhead increases significantly due to route failure and repair notification. For this reason, there has been a growing acceptance that the traditional purely greedy forwarding approaches are not optimal in most practical settings where the unit disk assumption or a perfect reception-within-range does not hold true. Some link-aware routing schemes have been recently reported [19-21]. However, the trade-offs between greediness and link quality has not been thoroughly studied. Furthermore, high mobility shortens the link duration between vehicles in the vicinity and might lead to performance degradation of the network. Therefore, in packet forwarding link life time should be considered to give higher priority to a candidate node which has higher link duration with the packet carrier node.

Moreover, the nature of traffic distribution in vehicular environments is heterogeneous (sparse and dense). In dense environments, routing protocols suffer from high over head due to proactive hello message broadcasting. The transmission of periodic "hello" messages under increased congested network consume resources, which can significantly affect the performance of VANET routing protocols on road segments especially during peek working hours in urban environment. Figure 2 illustrates this problem with the help of simple city scenario. The source node $S$ broadcasts "hello" messages to direct neighbors to find routes for destination node $D$. Flooding may be required to get updated routing information. Each neighbor node transmits its own information including location and IP address to neighboring nodes. If there are only a few nodes on road segments, the messages can easily be forwarded to next hops within a short time. However, in case of traffic congestion, the average delay significantly increases because each node is transmitting information at the same time.

The greedy forwarding mode of geographical routing protocols such as GPSR [4], GDBF [16], GPSR +AGF [22], GRANT [23] handle traffic congestion in such a way that the neighbor node which is closest to destination node is selected to forwarded messages. For example, as shown in the hatched area in Figure 2, node $N_{1}$ forwards a message to $N_{2}$ as $N_{2}$ is the shortest distance 
from destination node $D$. In the first transmission, the total number of nodes transmitting "hello" messages with each other is nine and each node also maintains it's routing table before forwarding this message to the next available node. $N_{2}$ forwards the message to destination node via $N_{3}$ and $N_{4}$. Finally, the source node $S$ establishes the route for destination node $D$ through nodes $N_{1} \rightarrow N_{2} \rightarrow N_{3} \rightarrow N_{4}$. The routing table of each node is updated every time they receive new messages in a congested network. As a result, the performance of packet delivery ratio and average delay are significantly affected.

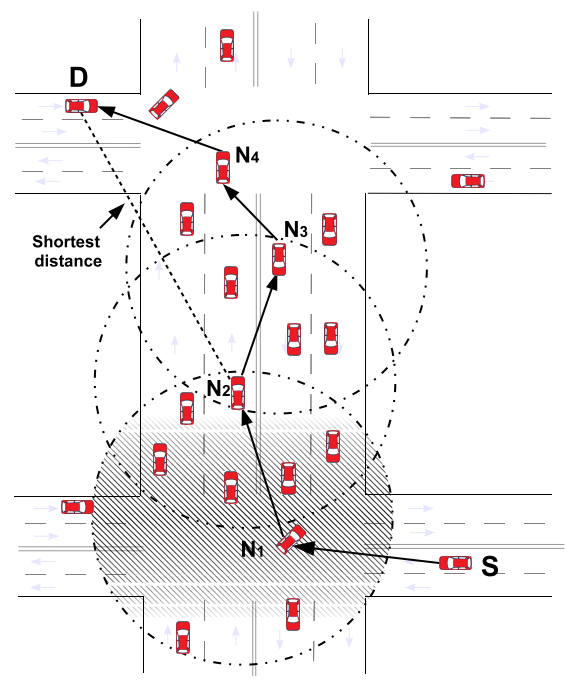

Figure 2: Traffic congestion problem in city scenario

In this paper, we have proposed a Multi-criteria Receiver Self-Election (MRSE) scheme to tackle the issues of high mobility and traffic congestion by suppressing the "hello" message and giving packet forwarding decision to the candidate receivers. In self-election process, a multicriteria waiting function uses four key parameters such as link life time, optimal distance from sender to receiver, optimal transmission range, and received power to determine the best next hop from all neighbors nodes. We assign the different weight values dynamically to each parameter and the next hops will use the same values of these parameters. It works as, the greater weight value has more impact than the parameter has in the self-election scheme. In previous schemes $[14,15,24]$, the static values are used for these factors. However, we have determined and adjusted the weight values according to the local traffic density information. This information is determined by calculating the number of nodes within the communication range of transmitter. The MRSE allows for the transfer of data packets quickly between intersections (streets) which significantly improve routing performance.

The rest of this paper is organized as follows, Section 2 presents the proposed multi-criteria receiver self-election scheme, its design, an example of an urban VANET scenario, and optimization. In Section 3, after describing an evaluation methodology, we present the performance analysis of the proposed scheme with two related receiver self-election and source selection schemes. The paper is concluded in Section 4.

\section{Proposed Multi-criteria Receiver Self-Election Scheme}

In this section, we present Multi-criteria Receiver Self-Election (MRSE) Scheme to determine the best next hop from all potential candidates. MRSE is a distributed process where the next relaying node is selected using four criteria including link life time, optimal distance, optimal 
transmission range and received power for non-uniform radio propagation in vehicular networks. We used IEEE 802.11 DCF (distributed coordination function) RTS/CTS (request-to-send/clearto-send) frames [25] to select a best next hop with less overhead.

\subsection{Receiver Self-Election using RTS/CTS}

It has already been discussed in literature that the route breaks due to high mobility and the network becomes congested through the frequent sending of "hello" messages. Our proposed MRSE scheme is based on a receiver side relay election approaches [13-16,24] that selects alternative nodes to handle route breaks caused by high mobility and implicitly eliminates the overhead by frequently sending "hello" messages. First, sender node broadcasts the RTS frame including the positions of the sender and destination nodes to all neighbors. Each receiving node calculates a waiting time, and this waiting time is sent back as a reply the CTS frame to the sending node. A waiting time assigned to each node basically determines how close to perfect this node is as best next node. The assignment of waiting time depends on the multi-criteria parameters that we will explain in Section 2.2. The node with the shorter waiting time will be considered as best node and will answer first by replying CTS to sender node. In the next step, the sender starts forwarding the data packets and receiver node acknowledge the data by sending ACK frame. Figures 3(a)-3(d) illustrate the whole procedure with the help of city scenario.

In Figure 3(a), node $N$ receives a message from source node $S$ for destination node $D$, looking to forward a message to the best next hop. This node broadcasts an RTS frame including its current position, the position of the destination node, and the transmission time of the RTS frame. The neighbor nodes calculate their waiting time to reply to node $N$ by sending a CTS frame once they receive the RTS frame. The nodes that are farther from the destination node than the sender are not involved in this process, for example node $N_{4}$ in Figure 3(a). Node $N_{1}$ is closest to the destination and has a shorter waiting time $(0.007 \mathrm{~ms})$, thus it replies with CTS first to node $N$. Nodes $N_{2}$ and $N_{3}$ will automatically cancel their timers when they overhear the CTS from $N_{1}$. Furthermore, the neighbor nodes of $N_{1}$, which are $A$ and $B$, will not send any messages before the transmission is completed. The neighbor list updates accordingly, if any node moves out of the communication range of node $N$. Node $N$ starts sending data packets after receiving the CTS from $N_{1}$. At the same time, the neighbor nodes (i.e., $N_{2}, N_{3}$ ) of $N$ will not send any messages until $N_{1}$ finishes sending the ACK frame to $N$. In this example, we have

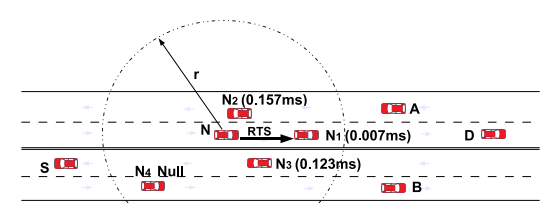

(a) Broadcasting RTS frame to all neighbors

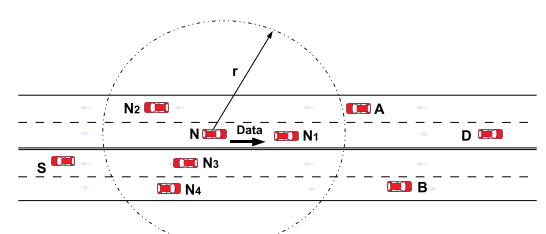

(c) Sending Data packets

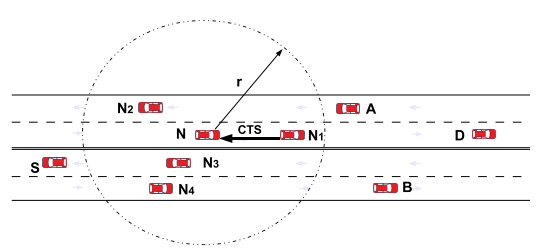

(b) Reply as CTS frame

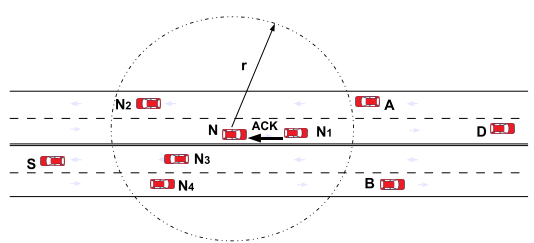

(d) ACK frame

Figure 3: Multi-criteria receiver self-election example 
illustrated how the best next hop can be effectively selected without sending "hello" messages in forwarding method.

The following is the proposed MRSE algorithm using multi-criteria parameters. In this algorithm, we have used three types of times such as (1) $\Delta_{R T S}, \Delta_{C T S}, \Delta_{D A T A}$, and $\Delta_{A C K}$ shows time to transmit RTS, CTS, DATA, and ACK frame; (2) $\tau_{i}$ shows waiting time of a node node $n_{i}$; and (3) Time to Live (TTL) of data packet. Initially, sender node $n_{c}$ looking for the next hop broadcasts a very short RTS frame including its positions and position of destination node. Each neighbor node call waiting function and calculate its waiting time $\tau_{i}$ which is set using weight values according to local density information. If a node is still receiving data packet then postpone transmission for DATA and RTS frame. A node which has less waiting time will first send-back a CTS frame to node $n_{c}$ and other node automatically discard their timers. After receiving CTS frame, the node $n_{c}$ will send DATA to best next node. Finally, current node will send ACK frame to complete transmission. This step continues until the data packet will reach at destination node.

\section{Notations:}

$\Delta_{R T S}, \Delta_{C T S}, \Delta_{D A T A}, \Delta_{A C K}:$ time to transmit for RTS, CTS, DAT A, and $A C K$ frame

$\tau_{i}=$ waiting time of node $n_{i}$

$p_{i}=$ position of node $n_{i}$

$p_{d}=$ position of the destination node

$n_{c}=$ current node ID that finds next hop

$T_{-} F L A G=$ Transmision status, (True/False)

\section{Proposed MRSE Algorithm:}

1: Initialy $T_{-} F L A G$ set to True

$2: \operatorname{if}\left(T_{-} F L A G \&\right.$ Receiving $R T S\left(p_{i}, p_{d}, \Delta_{D A T A}\right)$ from node $n_{c}$ then

3: Call waiting function and calculate $\tau_{i}$

4: Set timer to $\tau_{i}$ using weight values according to local density information

$5: \quad$ if(RTS frame transmission complete) then

6: $\quad$ Set $T \_F L A G$ to True

7: else

8: $\quad$ Postpone transmissions for $\Delta_{D A T A}+\Delta_{R T S}$

9: $\quad$ Set $T_{-} F L A G$ to False

10: end if

11 : else

$12:$ if $\left(T_{-} F L A G\right.$ \& Receiving $C T S\left(n_{j}, n_{c}, \Delta_{D A T A}\right)$ from node $n_{j}$ before the timeout) then

13: Cancel timer $/ * n_{j}$ is the best next hop candidate $*$ /

14: if(CTS frame transmission complete) then

15: $\quad$ Set $T_{-} F L A G$ to True

16: else 
17: $\quad$ Postpone transmissions for $\Delta_{D A T A}$

18: $\quad$ Set $T_{-} F L A G$ to False

19: end if

20 : else

21 : if $\left(T_{-} F L A G \&\right.$ Overhearing $D A T A$ from node $\left.n_{c}\right)$ then

22: if(DATA transmission complete) then

23: $\quad$ Set $T$ FLAG to True

24: else

25: $\quad$ Postpone transmissions for $\triangle_{A C K}$

26: $\quad$ Set $T_{-} F L A G$ to False

27: $\quad$ end if

28 : end if

29 : Upon timout :

30 : Broadcast $C T S\left(n_{i}, n_{c}, \Delta_{D A T A}\right) / * n_{i}$ is the best next node $* /$

\subsection{Multi-Criteria Waiting Function}

In this section, we illustrate how the waiting time is determined which is based on multicriteria waiting function to select the best next hop. In multi-criteria waiting function, we try to achieve the following objectives:

1. The best next hop should reply first with shortest time.

2. To avoid time collisions of second best next hop, the waiting time difference should be reasonable.

3. The waiting time should not be too long that creates unnecessary delays.

Four key parameters, including link life time, optimal distance from sender to receiver, optimal transmission range, and received power are used to achieve these goals. Finally, different weights are assigned and dynamically adjusted based on the traffic and network conditions.

\section{Link Life Time}

The link life time estimates the link quality of the wireless channels between each vehicle. This duration represents the minimum time in which two direct neighbor vehicles can exchange information with guaranteed delivery. Since vehicles are travelling at high speed, this time interval can be very short. Therefore, we place a great deal of reliance on this time interval to give higher priority to those nodes whose connection time lasts longer. We consider the distance between two nodes as the distance between two vectors rather than the distance between two static points. The position vector of $\vec{p}_{i}$ of each node $N_{i}$ is defined by the following equation:

$$
\overrightarrow{p_{i}}=\left(x_{i}+\nu_{x} t, y_{i}+\nu_{y} t\right)
$$

where $t$ is the time since the initial position and speed vector of node $N_{i}$ were $\vec{p}_{i}=\left(x_{i}, y_{i}\right)$ and $\overrightarrow{\nu_{i}}=\left(\nu_{i x}, \nu_{i y}\right)$ respectively. The function of the distance square $\delta^{2}(t)$ is defined as:

$$
\delta^{2}(t)=\left(\overrightarrow{p_{1 x}}-\overrightarrow{p_{2 x}}\right)^{2}+\left(\overrightarrow{p_{1 y}}-\overrightarrow{p_{2 y}}\right)^{2}
$$




$$
\delta^{2}(t)=\left[\left(x_{1}-x_{2}\right)+t\left(\nu_{1 x}-\nu_{2 x}\right)\right]^{2}+\left[\left(y_{1}-y_{2}\right)+t\left(\nu_{1 y}-\nu_{2 y}\right)\right]^{2}
$$

Suppose $\lambda_{x}=\left(x_{1}-x_{2}\right), \lambda_{y}=\left(y_{1}-y_{2}\right), \lambda_{s x}=\left(\nu_{1 x}-\nu_{2 x}\right), \lambda_{s y}=\left(\nu_{1 y}-\nu_{2 y}\right)$, by putting these values in Equation 3, we have the simplified form of distance function $\delta^{2}(t)$ as follows:

$$
\delta^{2}(t)=\left(\lambda_{x}^{2}+\lambda_{y}^{2}\right)+t^{2}\left(\lambda_{s x}^{2}+\lambda_{s y}^{2}\right)+2 t\left(\lambda_{x} \lambda_{s x}+\lambda_{y} \lambda_{s y}\right)
$$

The Equation 4 is second degree polynomial that only assumes non-negative values. Therefore, the smallest value of $\delta^{2}(t)$ occurs when its derivative $\delta^{2 \prime}(t)$ equals to zero and the value of $t$ is determined as:

$$
\bar{t}=\frac{-\left(\lambda_{x} \lambda_{s x}+\lambda_{y} \lambda_{s y}\right)}{\left(\lambda_{s x}^{2}+\lambda_{s y}^{2}\right)}
$$

The $\bar{t}$ gives the two types of connection times, if the value of $\bar{t}$ is positive then the nodes are getting closer to each other; if negative, the nodes are moving away from each other. We calculate the link life time until the node goes out of communication range.

\section{Optimal Distance from Sender to Receiver}

This parameter determines the optimal distance between a sender node $S$ and intermediate node $N_{i}$ for destination node $D$. A single criteria receiver election schemes $[13,14,17]$ usually used this parameter. The optimal distance is defined as follows:

$$
d_{i}=d_{S D}-d_{N_{i} D}
$$

where $d_{i}, d_{S D}$, and $d_{N_{i} D}$ are the distances between nodes $S-N_{i}, S-D$, and $N_{i}-D$ respectively. These distances actually denote the progression towards the destination node, if node $N_{i}$ is the next hop and closest to the destination.

\section{Optimal Transmission Range}

The optimal transmission range $f_{i}$ of a node $N_{i}$ describes the probability that the data packet is successfully received by a node. Wireless channels are error-prone and do not provide any guarantee that signals out-side of particular range will successfully transmitted. There are many factors that may obstruct the radio signals. For example, a node away from the nominal range may receive a RTS frame but may not receive data packets successfully. This problem happen in real wireless radios channels because it does not follow unit disk assumption [26].

To find the optimal transmission range, we use a translation function proposed by [24]. In this function, the distance from the sender node is used as an input and the optimal transmission range is the output. For example

$$
f_{\text {trans }}(x)=\left\{\begin{array}{l}
x+R_{t d} \quad \text { if } x \leq R_{o t} \\
-x+R_{\max } \quad \text { if } x>R_{o t}
\end{array}\right.
$$

where $R_{o t}$ shows the optimal transmission range of a sender node, $R_{\max }$ the estimated maximum transmission range with acceptable error rate, and $R_{t d}$ is the translation distance. For more dynamic results, these parameters can be set according to the network conditions in the area. 


\section{Received Power}

The received power $p_{i}$ of sender node provides signals with real channel quality. Many researchers are trying to calculate the optimal transmission range with received power. However, different obstacles such as big buildings, trees, advertisement boards, traffic lights, etc may block the radio signals in the real-life deployment of V2V and V2I communications. The received power at a particular node can differentiate the nodes at comparable distances. The reasoning is that if the vehicle is moving, the quality of the reported data is not affected by the received signal power; however the real reason is that the distance travelled by a vehicle is negligible when it receives the RTS frame.

Finally, the multi-variable function is used and customize it into a four variable polynomial of the selected parameters. The waiting time of any node $t_{i}$ returned by this function within the time interval $\left[0, T_{\max }\right]$ (where $T_{\max }$ is the maximum waiting time) is determined as:

$$
f\left(\bar{t}_{i}, d_{i}, d_{S N_{i}}, p_{i}\right)=A \bar{t}_{i}^{w_{1}} d_{i}^{w_{2}} f_{i}^{w_{3}} p_{i}^{w_{4}}+T_{\max }
$$

where $A=\frac{-T_{\max }}{\bar{t}_{\max }^{w_{1}} f_{\max }^{w_{2}} f_{\max }^{w_{3}} p_{\max }^{w_{4}}}$ and $w_{i}(i=1,2,3,4)$ are the weights of each parameter. The weight values of these parameters are adjusted using the mapping function proposed by [14]. In mapping function, the main objective is to compute a single ranking scale through the use of an aggregating function that weighs all criteria into a single unit. We assign the different weight values dynamically to each parameter and the next hops will use the same values of these parameters. It works as, the greater weight value has more impact than the parameter has in the self-election scheme. In previous schemes [14,24], the static values are used for these factors. However, we have determined and adjusted the weight values according to the local traffic density information. Each node independently computes the weight values according to the number of vehicles within its radio coverage. This local traffic density information is estimated based upon each node's Contention Window (CW). According to the Ke, et al. [27] the value of $\mathrm{CW}$ is higher in heavy traffic density, which means that frequent retransmission occurs between contending nodes due to an increase of the probability of collision. On the contrary, lower CW implies that the light traffic density. Accordingly, when vehicles within the communication range of the source receives RTS frame, they simply check their $\mathrm{CW}$ in order to dynamically adjust the weight values. It is note worthy that this method of dynamic weight adjustment does not generate any network and computational overhead.

\subsection{Impact of Multi-criteria Waiting Function}

The main purpose of using multi-criteria waiting function is to determine a single value based on the above mentioned key parameters. An optimal decision is taken based on this final single value. The impact of multi-criteria waiting function is compared with forwarding progress only, as shown in Figures 4 and 5. The waiting times are determined when vehicles receive an RTS frame request between transmitter at position $(0,0)$ and destination at position $(600,200)$. As Figure 4 shows the forward progress without multi-criteria also determined the waiting time out of the transmission range that may cause the loss of many data packets. The waiting time value is around 0.9 seconds and the transmission range is more than 400 meters in this case.

The Figure 5 shows the nodes waiting time using proposed MRSE when an RTS frame is received from different positions near the transmitter. We adjusted weight values $w_{1}, w_{2}, w_{3}$, and $w_{4}$ according to the network condition in multi-criteria waiting functions, in this example the weight values are $w_{1}=0.45, w_{2}=0.3, w_{3}=1.4$, and $w_{4}=0.02$. We considered the optimal transmission rage to be $300 \mathrm{~m}$. However, we did not assume perfect reception due to interference generated by the urban environment. We used a Shadowing propagation model proposed by [28] 


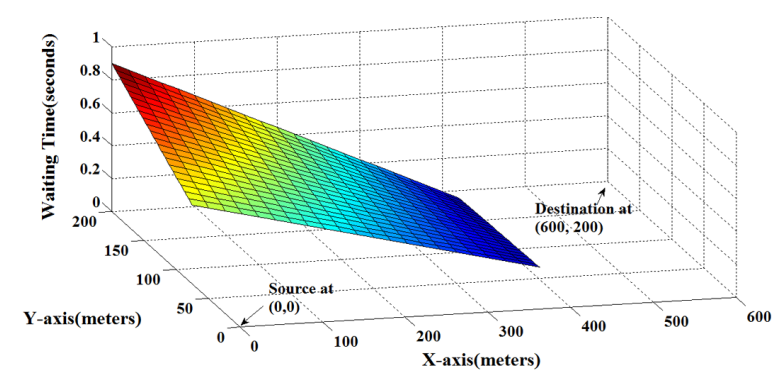

Figure 4: Waiting time using forwarding progress only

to calculate the received power. The Figure 5 illustrates the shortest waiting time and optimal transmission range using the proposed MRSE scheme with four key parameters. In case of four criteria waiting function, the waiting time is around 0.5 seconds within transmission of range 285 meters. The results show that the proposed MRSE prefers nodes with optimal transmission range and shortest waiting time as compared to forward progress method only.

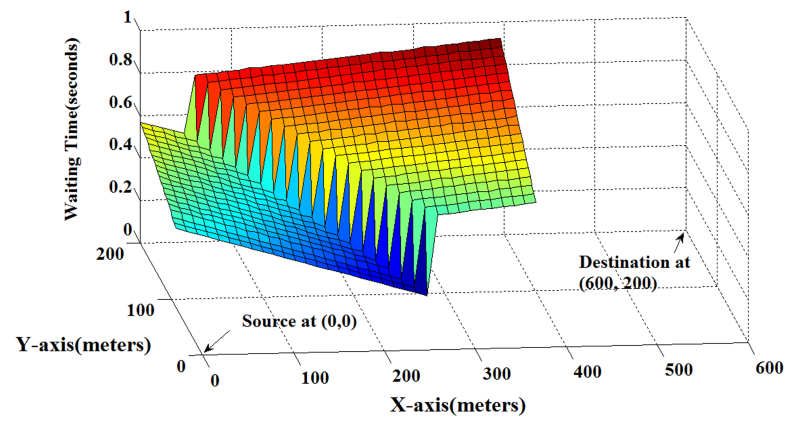

Figure 5: Waiting time using four criteria (link life time, optimal distance from sender to receiver, optimal transmission range, and received power) waiting function

\section{Performance Evaluation}

This section presents a detailed analysis of the proposed MRSE scheme applied to our recently proposed Reactive Traffic-Aware Routing Strategy (ReTARS) [29] for real-time urban vehicular environments. The MRSE scheme is used to reduce traffic overhead in the streets, helping to transfer data packets quickly. ReTARS leverages prior global knowledge of real-time vehicular traffic to create paths between each vehicle. In ReTARS, the critical decisions are taken at the road intersections where the decision making node evaluates the best possible routes towards the destination based on the prior global knowledge of real-time vehicular traffic. ReTARS is fairly well-understood and can be used in this domain because it accommodates the frequent network disconnections and traffic congestion that are observed in many vehicular networks.

All experiments are conducted on a map of Chicago city with simulation dimension $3968 \mathrm{~m} \times 1251 \mathrm{~m}$. The area contains 370 road segments with a total length of 3630394.0 meters extracted from TIGER/LINE line databases [30]. The main reason for the big area is to test the performance of MRSE in high mobility and increased traffic congestion. The integrated, configurable, and scalable Swans ++ simulator [31] with IEEE.11b DCF standard is used to evaluate the performance of the proposed MRSE scheme. To generate and evaluate a large number of different scenarios, the numbers of vehicles and data packet rates are varied for with obstacles in urban environ- 
Table 1: Parameter values used in simulation for proposed MRSE scheme

\begin{tabular}{|c|c|}
\hline Parameter & Value \\
\hline Simulation dimension & $3968 \mathrm{~m} \times 1251 \mathrm{~m}$ \\
\hline Simulation area & $3630394.0 \mathrm{~m}$ \\
\hline Number of vehicles & $150,250,350$ \\
\hline Warning packet size & 512 bytes \\
\hline Normal packet size & 1024 bytes \\
\hline Packet sending frequency & 1 per second \\
\hline Transmission range & $400 \mathrm{~m}$ \\
\hline Simulation time & $400 \mathrm{~s}$ \\
\hline Vehicle speed & $20-80 \mathrm{~m} / \mathrm{h}$ \\
\hline Mobility model & STRAW \\
\hline Routing protocol & ReTARS \\
\hline MAC protocol & IEEE $802.11 \mathrm{DCF}$ \\
\hline
\end{tabular}

ment. The total simulation time for a single flow was 400 seconds which is a reasonable time for this area of the map and the number of nodes. However, to obtain a more accurate result, the first 100 seconds of simulation are discarded. The STreet RAndom Waypoint (STRAW) mobility model [32] is used for node mobility. The nodes were placed on the map using the random placement model and experiment was repeated for 15 flows. In each experiment 10 source and destination nodes pairs with different CBR and UDP packets are selected randomly. In the proposed MRSE scheme, the packet carrier node needs to have both self and destination locations for packet forwarding purposes. To gain access of such location information, we utilized the implemented scalable and distributed location service in Swans ++ packet level network simulator. Similarly, each node can compute its self direction and speed vectors by using the implemented street mobility module in Swans ++ network simulator. In MRSE, each node in the radio coverage of the packet carrier node calls the waiting function when it receives a modified RTS frame. When a node receives a modified RTS frame, waiting function is called to compute waiting time for the selection of best next hop. This setting helps to get more accurate neighbor information. Table 1 describes the simulation parameters used in all experiments.

\subsection{Simulation Results in Urban Environment (With Obstacles Scenario)}

The performance of the proposed MRSE scheme is evaluated using PDR and average delay by varying the number of vehicles for with obstacles urban scenario. Figures 6(a)-6(c) show the simulation results that determine the PDR of proposed MRSE method by comparing these results with the two most related receiver self-election schemes [14,24] and source-selection scheme that periodically send "hello" packets to all neighbors. A slight modification of IEEE 802.11 (with DCF standard) RTC/CST frame is used to select the best next hop using receiver self-election scheme. As shown in Figures 6(a)-6(c), the PDRs of proposed MRSE scheme steadily increased from $65 \%$ to $81 \%$ when node density increased from 150 to 350 nodes.

The PDR of proposed MRSE scheme is consistently higher than Nzouonta et al., Egoh \& De, and source-selection schemes in all cases. This is because the weight values were carefully assigned to each parameter to compute waiting time. The PDR of Nzouonta et al., self-election scheme is about $8 \%$ to $11 \%$ lower than MRSE in all cases. The main reason for the low results of Nzouonta et al., scheme is the assignment of static weights values to determine a waiting time using multi-criteria waiting function. The PDR of Egoh \& De self-election scheme is apparently lower than MRSE, which is about $15 \%$ when node density is 150, (Figure 6(a)) and $20 \%$ in case of 


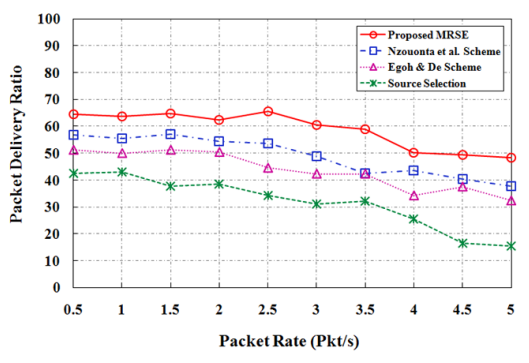

(a) PDR using 150 nodes

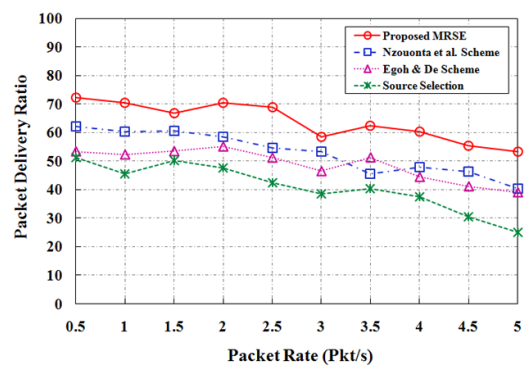

(b) PDR using 250 nodes

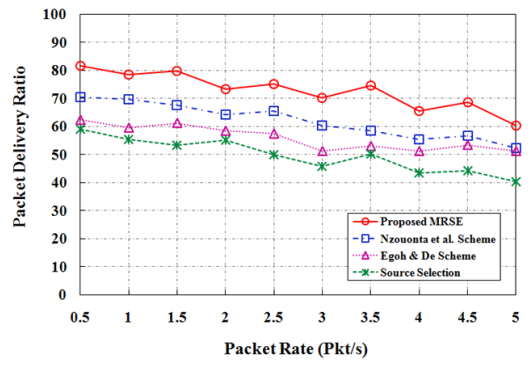

(c) PDR using 350 nodes

Figure 6: Packet delivery ratio for proposed MRSE, Nzouonta et al., [24], Egoh \& De [14], and Source-Selection schemes for with obstacles scenario

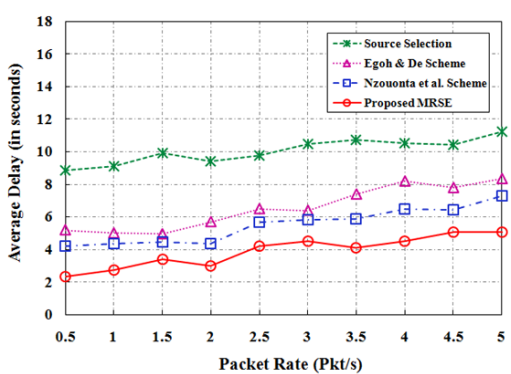

(a) Average delay using 150 nodes

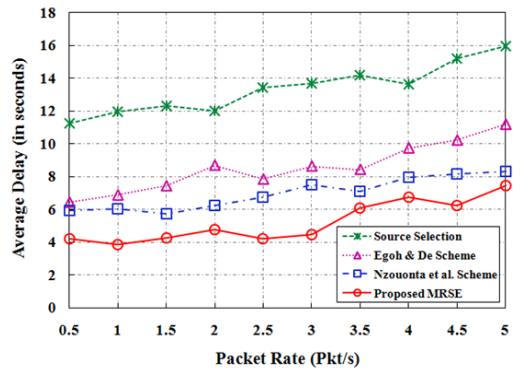

(b) Average delay using 250 nodes

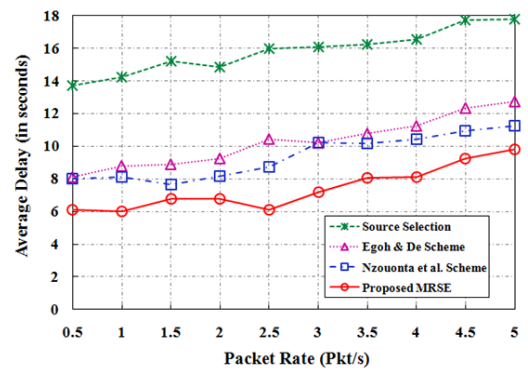

(c) Average delay using 350 nodes

Figure 7: Average delay for proposed MRSE, Nzouonta et al., [24], Egoh \& De [14], and SourceSelection schemes for with obstacles scenario

high density, as depicted in Figures 6(b) and 6(c). This is because two criteria based forwarding node selections, hop progress (greediness) and reachability (link quality) were used to determine waiting time to select best next hop. Similarly, the PDR of source selection method is lower than other protocols, as even the PDR starts falling below $50 \%$ when the CBR is just around 2 
packets rate/sec. This is due to the frequent broadcast of "hello" messages that each node needs to create and to maintain a list of neighbors. The overall PDR for all schemes are not so high, as was expected. The implementation of more accurate RPM with obstacles that reduces the contention level is the main reason for this low percentage of PDR.

The average delay of proposed MRSE, Nzouonta et al., [24], Egoh \& De [14], and sourceselection schemes are shown in Figures $7(\mathrm{a})-7(\mathrm{c})$. The average delay of the proposed MRSE is consistently lower than other protocols in all cases. The average delay of Nzouonta et al., receiver self-election scheme is slightly higher than our proposed MRSE, as even node densities increased from 150 to 350 nodes. For comparison, Egoh \& De receiver-self election scheme has around 7-12 seconds average delay when node densities increase from 150 to 350 nodes, which is about 5 seconds higher than MRSE. Source selection schemes have three times higher delay as compared to MRSE. The main reason for the better performance of MRSE is the careful adjustment of weight values to each selection parameter that selects the best next hop candidate within the shortest time to avoid unnecessary delays. As a result, better link utilization is received for data transfer. Also, it leads to improved delays as it needs less retransmission and backoffs.

\section{Conclusions}

In this paper, a multi-criteria receiver self-election (MRSE) scheme is proposed to suppress the sending of periodic "hello" messages that significantly degrade the network performance of end-to-end data transfer rates. The waiting function is defined using multi-criteria parameters to select best next hop. Four key parameters are used including link life time, optimal distance from sender to receiver, optimal transmission range, and received power. The simulation results using packet delivery ratio and average delay show that the proposed scheme offer better performance as compared to two receiver self-election using RTS/CTS-based schemes and source selection using "hello" packet scheme. The proposed MRSE scheme forwards data between intersections (streets) and it performs better in real time urban environments where large obstacles such as buildings may block radio signals. These results show that distributed applications that generate moderate or high traffic can be successfully implemented in VANETs.

\section{Bibliography}

[1] C.E. Perkins and E.M. Royer, Ad-hoc on-demand distance vector routing, Proc.of the Second IEEE Workshop on Mobile Computer Systems and Applications, pp.90-100, 1999

[2] David B. Johnson and David A. Maltz, Dynamic Source Routing in Ad Hoc Wireless Networks, Kluwer Academic Publishers, 1996.

[3] P. Jacquet, P. Muhlethaler, T. Clausen, A. Laouiti, A. Qayyum, and L. Viennot, Optimized link state routing protocol for ad hoc networks, Technical report, HIPERCOM Projet, INRIA Rocquencourt, 62-68, 2001.

[4] Brad Karp and H. T. Kung, Gpsr: greedy perimeter stateless routing for wireless networks. In MobiCom '00: Proc. of the 6th annual int. conf. on Mobile computing and networking, New York, NY, USA, 243-254, 2000.

[5] Prosenjit Bose, Pat Morin, Ivan Stojmenovic, and Jorge Urrutia, Routing with guaranteed delivery in ad hoc wireless networks, Wireless Networks, 7(6):609-616, 2001. 
[6] Fabian Kuhn, Roger Wattenhofer, Yan Zhang, and Aaron Zollinger, Geometric ad-hoc routing: of theory and practice, In PODC '03: Proc. of the twenty-second annual symposium on Principles of distributed computing,New York, NY, USA, 63-72, 2003.

[7] Christian Lochert, Martin Mauve, Holger Fussler, and Hannes Hartenstein, Geographic routing in city scenarios, SIGMOBILE Mob. Comput. Commun. Rev., 9:69-72, January 2005.

[8] K.C. Lee, J. Haerri, Uichin Lee, and M. Gerla, Enhanced perimeter routing for geographic forwarding protocols in urban vehicular scenarios. In Globecom Workshops, 2007 IEEE, 1-10, 2007.

[9] Kevin C. Lee, Pei-Chun Cheng, and Mario Gerla, Geocross: A geographic routing protocol in the presence of loops in urban scenarios, Ad Hoc Networks, 8(5):474-488, 2010.

[10] R. Khokhar, R. Md Noor, K. Ghafoor, C-H Ke, and N. Md Asri. Fuzzy-assisted social-based routing for urban vehicular environments, EURASIP Journal on Wireless Communications and Networking, 2011(1):178, 2011.

[11] C. Lochert, H. Hartenstein, J. Tian, H. Fussler, D. Hermann, and M. Mauve, A routing strategy for vehicular ad hoc networks in city environments, Intelligent Vehicles Symposium, 2003. Proceedings. IEEE, 156-161, 2003.

[12] Tonghong Li, S.K. Hazra, and W. Seah, A position-based routing protocol for metropolitan bus networks, Vehicular Technology Conference, 2005. VTC 2005-Spring. 2005 IEEE 61st, 4:2315-2319, 2005.

[13] Holger Fubler, Jrg Widmer, Michael Ksemann, Martin Mauve, and Hannes Hartenstein, Contention-based forwarding for mobile ad hoc networks, Ad Hoc Networks, 1(4):351-369, 2003.

[14] K. Egoh and S. De. A multi-criteria receiver-side relay election approach in wireless ad hoc networks. In Military Communications Conference, MILCOM 2006, IEEE, 1-7, 2006.

[15] Komlan Egoh and Swades De, Priority-based receiver-side relay election in wireless ad hoc sensor networks. In IWCMC '06: Proc. of the 2006 int. conf.on Wireless communications and mobile computing, New York, NY, USA, 1177-1182, 2006

[16] Mohit Chawla, Nishith Goel, Kalai Kalaichelvan, Amiya Nayak, and Ivan Stojmenovic, Beaconless position based routing with guaranteed delivery for wireless ad-hoc and sensor networks. In Ad-Hoc Networking, IFIP International Federation for Information Processing, Springer Boston,212:61-70. 2006.

[17] M. Zorzi and R.R. Rao, Geographic random forwarding (geraf) for ad hoc and sensor networks: multihop performance, Mobile Computing, IEEE Transactions on, 2(4):337-348, oct. 2003.

[18] S. De, On hop count and euclidean distance in greedy forwarding in wireless ad hoc networks. Communications Letters, IEEE, 9(11):1000-1002, 2005.

[19] Karim Seada, Marco Zuniga, Ahmed Helmy, and Bhaskar Krishnamachari, Energy-efficient forwarding strategies for geographic routing in lossy wireless sensor networks. In Proc. of the 2nd int. conf. on Embedded networked sensor systems, SenSys '04, 108-121, 2004. 
[20] Seungjoon Lee, Bobby Bhattacharjee, and Suman Banerjee, Efficient geographic routing in multihop wireless networks. In Proc. of the 6th ACM int. symposium on Mobile ad hoc networking and computing, MobiHoc '05, New York, NY, USA, 230-241, 2005.

[21] M.R. Souryal and N. Moayeri, Channel-adaptive relaying in mobile ad hoc networks with fading, In Sensor and Ad Hoc Communications and Networks, 2005. IEEE SECON 2005. 2005 Second Annual IEEE Communications Society Conference on, 142-152, 2005.

[22] Valery Naumov, Rainer Baumann, and Thomas Gross, An evaluation of inter-vehicle ad hoc networks based on realistic vehicular traces, In MobiHoc '06: Proceedings of the 7th ACM international symposium on Mobile ad hoc networking and computing, New York, NY, USA, 108-119, 2006.

[23] S. Schnaufer and W. Effelsberg, Position-based unicast routing for city scenarios. In World of Wireless, Mobile and Multimedia Networks, 2008 International Symposium on a, 1-8, 2008.

[24] J. Nzouonta, N. Rajgure, Guiling Wang, and C. Borcea, Vanet routing on city roads using real-time vehicular traffic information. Vehicular Technology, IEEE Transactions on, 58(7):3609-3626, 2009.

[25] The Institute of Electrical and Electronic Engineers (IEEE), Wireless lan medium access control (mac) and physical layer scpecifications.

[26] Gang Zhou, Tian He, Sudha Krishnamurthy, and John A. Stankovic, Impact of radio irregularity on wireless sensor networks, In MobiSys '04: Proceedings of the 2nd international conference on Mobile systems, applications, and services, 125-138, 2004.

[27] Chih-Heng Ke, Chih-Cheng Wei, Kawuu W. Lin, and Jen-Wen Ding, A smart exponentialthreshold- linear backoff mechanism for ieee 802.11 wlans, Int. J. of Communication Systems, 24(8):1033-1048, 2011.

[28] T. S. Rappaport, Wireless Communications Principles and Practice, Prentice Hall, 2nd edition edition, 2002.

[29] R. Khokhar, A. Ngadi, M. S. Latiff, and M. A. Amin, Reactive traffic-aware routing strategy for urban vehicular environments, International Journal of Ad Hoc and Ubiquitous Computing, 10(3):149-163, 2011.

[30] Tiger. tiger/line and tiger-related products. u.s. census bureau, 2011.

[31] Swans ++ . Swans ++ - extensions to the scalable wireless ad-hoc network simulator, 2011.

[32] David R. Choffnes and Fabian E. Bustamante. An integrated mobility and traffic model for vehicular wireless networks. In VANET '05: Proceedings of the 2nd ACM international workshop on Vehicular ad hoc networks, New York, NY, USA, 69-78, 2005. 